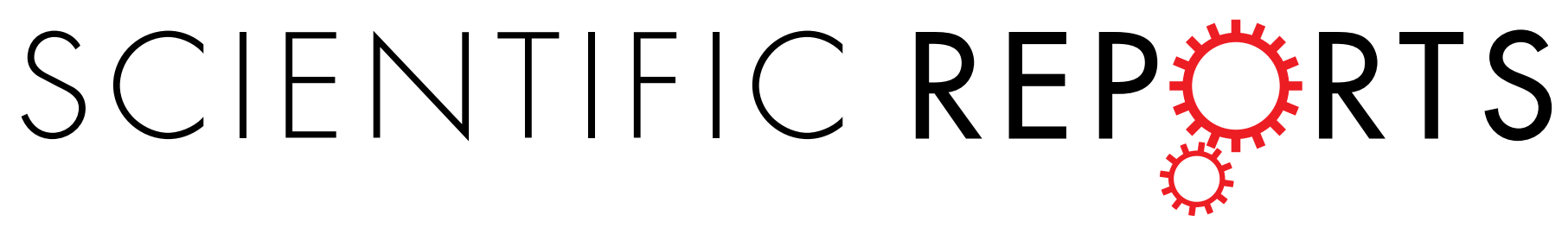

\title{
Corrigendum: Preferential enhancement of laser-driven carbon ion acceleration from optimized nanostructured surfaces
}

Malay Dalui, W.-M. Wang, T. Madhu Trivikram, Subhrangsu Sarkar, Sheroy Tata, J. Jha, P. Ayyub, Z. M. Sheng \& M. Krishnamurthy

Scientific Reports 5:11930; doi: 10.1038/srep11930; published online 08 July 2015; updated 27 August 2015

The original version of this Article contained a typographical error in the spelling of the author Subhrangsu Sarkar which was incorrectly given as Subhrangshu Sarkar. This has now been corrected in the PDF and HTML versions of the Article. 